\title{
Une Étude critique de Madame la présidente de Fatou Fanny-Cissé
}

\author{
Ngozi Obiajulum Iloh ${ }^{1}$
}

Accepted: 22 December 2020 / Published online: 6 April 2021

(C) The Author(s) 2021

\begin{abstract}
This article discusses the Ivorian writer, Fatou Fanny-Cissé's novel, Madame la présidente, published in 2015. The novel offers a fundamental critique of African democracy and the contemporary politics in Africa. The Republic of Louma is an imaginary country that show-cases electoral crises in an imaginary contemporary continent. The plot about a female dictator has a strong feminist inclination. The feminisation of presidential elections is a caricature of the dictatorial tendencies of African leaders. The themes discussed are true to contemporary political events in Africa as well as other parts of the world. The presentation reveals a lucid picture of feminine dictatorial politics.
\end{abstract}

Keywords Woman $\cdot$ Feminism $\cdot$ Female president $\cdot$ Politics $\cdot$ Power

\section{Résumé}

Dans cet article, il s'agit d'une étude critique du roman, Madame la présidente de l'Ivoirienne, Fatou Fanny-Cissé, publié en 2015. C'est un roman basé sur la critique de la démocratie africaine révélant la politique en Afrique contemporaine. Un pays imaginaire, la République Démocratique de Louma manifeste les crises électorales au continent contemporain. Un roman fortement féministe avec l'acclamation d'une présidente au pouvoir. C'est un féminisme mystique ou une dictature féministe et une politique dictatoriale féministe. La féminisation des élections présidentielles n'est qu'une caricature des tendances de la dictature des leaders africains. Les thèmes abordés sont vraisemblables au regard des événements politiques contemporains en Afrique ainsi que partout dans le monde. La lucidité de la politique dictatoriale au féminin ou la dictature féministe est présentée avec un mélange d'un certain mysticisme bien recherché. A ce pouvoir politique présidé par Fitina, s'entremêlent la corruption, le népotisme, l'association estudiantine et l'extention du pouvoir de la présidente, ce qui ne se distingue pas du pouvoir politique masculin. C'est une grande tragédie d'une assoiffée de pouvoir qui incarne presque tous les dirigeants africains

Ngozi Obiajulum Iloh

ngozi.iloh@uniben.edu; iloh214@gmail.com

1 Department of Foreign Languages, University of Benin, P.M.B. 1154, Benin City, Nigeria 
ainsi que des nouveaux leaders mondiaux. Tout a été brisé car, la présidente échoue, elle a manipulé ses amis et sa parenté.

Mots clés Femme $\cdot$ Féminisme $\cdot$ Féministe $\cdot$ Présidente $\cdot$ Politique $\cdot$ Pouvoir

\section{Introduction}

Fatou Fanny-Cissé, romancière Ivoirienne, publie en 2015 un roman contemporain intitulé Madame la présidente aux Editions NEI-CEDA à Abidjan. Madame la Présidente est un roman de 227 pages écrit en français. Le roman a gagné le « prix du meilleur livre de fiction de l'année- ALA $2017 »$ le 17 juin 2017. Enseignant-chercheur à l'Université Felix Houphouët-Boigny, Fatou Fanny-Cissé est née en 1971 en Côte d'Ivoire.

Dans le roman, la romancière énonce une variété de discours sur la politique en Afrique contemporaine. En prenant un pays imaginaire dénomé la république de Louma, elle démontre les crises électorales en Afrique contemporaine. Ce continent peut aussi représenter les pays en voie de développement.

Le roman est une grande tragédie d'une assoiffée de pouvoir qui incarne presque tous les dirigeants africains ainsi que des nouveaux leaders mondiaux. C'est un roman féministe écrit par une femme ayant un protagoniste principal féminin et un Premier ministre féminin ainsi qu'une présidente estudiantine. Tout s'est écrasé car, la présidente échoue, elle a manipulé ses amis et sa parenté. Elle se contamine les mains du sang des innocents et est abandonnée. Tous les protagonistes féminins échouent.

On se demande si ce n'est pas un moyen de cacher les méfaits des leaders africains qui sont des dictateurs, car la romancière doit se protéger des leaders tyraniques. C'est un roman vif, plein de langage simple et d'africanismes intéressants. C'est un nouveau roman contemporain qui ressemble à Le Cercle des tropiques d'Alioune Fantouré, le Guinéen; à La Victime d'Yves-Emmanuel Dogbé (1979) et à La Calebasse cassée de Tunde Fatunde (2002). Tous ces écrivains se servent de pays imaginaires qui ressemblent aux pays africains. Fantouré se sert de pseudonyme comme Fatou Fanny-Cissé. Tous ces écrivains ont un certain engagement littéraire dans leurs œuvres.

\section{Techniques narratologiques}

Roman hétérodiégétique avec une narration omnisciente, le roman est subdivisé en 15 chapitres de narration vivante et jalonnée de descriptions échauffées et brûlantes, la narratrice parsème sa narration des analepses et des possibles prolepses. Avec cette technique narratologique, on parvient à suivre les évènements romanesques d'une manière cinématographique. On arrive aussi à suivre la narratrice minutieusement partout où la focalisation se tourne en entrelaçant d'une manière embellisante les thèmes abordés. 


\section{Préoccupations thématiques}

Les thèmes abordés couvrent les évènements politiques contemporains en Afrique ainsi que partout dans le monde. C'est une critique de la démocratie loumaise qui incarne aussi la démocratie du continent inconnu mais que l'on soupçonne être la démocratie africaine. Une nouvelle démarche déborde avec une certaine lucidité de la politique dictatoriale au féminin ou la dictature féministe jalonnée par un mélange d'un certain mysticisme bien recherché. Les élections et les évènements pré-électoraux et tout ce qui caractérise les élections doivent être supportés au cours du gouvernement. Madame la présidente, Fitina, gouverne d'une main de fer et par la corruption. D'autres thèmes sont incorporés à la corruption, tels que le népotisme, l'exploitation de l'association estudiantine, l'injustice, l'extention du mandat de la présidente et les élections caractérisées par un grand mysticisme. L'injustice et le terrorisme caractérisent le gouvernement de Fitina. On peut comparer ces thèmes et son engagement politique à ceux de Fantouré (1972) également énoncés par Adejir (2000) et Elaho (2000). Au niveau du mysticisme où l'on parle du fantastique, Fanny-Cissé ressemble à Sony Labou Tansi dans son roman La Vie et demie où se trouvent des personnages surnaturels que Emokpae-Ogbebor (2017) a bien affirmé dans son article. Pour caractériser tous ces thèmes, la romancière se sert des personnages romanesques.

\section{Les personnages}

Le développement de la caractérisation chez Fanny-Cissé manque un peu de profondeur pour certains personnages. Nous reconnaissons trois classifications principales de personnages dans ce roman. On peut distinguer les personnages féminins, les personnages masculins et les personnages fantomatiques.

\section{Les personnages féminins}

Toutes les femmes et les filles constituent des personnages féminins. Parmi les personnages féminins se trouve la protagoniste principale qui est Madame la Présidente, Fitina. Fitina est l'aînée et la sœur de trois enfants orphelins. Elle est célibataire et sans enfant. Elle est la seule candidate parmi quarante candidats aux élections présidentielles de son pays, Louma. On ne connait rien de sa naissance, de son enfance et de son adolescence. Elle devient célèbre à cause des élections politiques dans son pays. Elle est une femme courageuse, forte et têtue. Elle est surnommée « femmemâle » et «femme-garçon » à cause de son courage (p. 23). Elle est une bonne harangueuse car elle est douée du 'verbe haut et flamboyant' (p. 25). Elle devient un personnage mystérieux à cause de son pouvoir assoiffé. Elle devient une grande 'dictateuse' ou plutôt dictateur. Le féminin de ce mot n'existe pas car les leaders féminins étaient aussi rares. Elle finit par éliminer tous ses ennemis avant qu'elle soit éliminée elle-même par les fantômes. Tous les autres personnages tournent autour de ce personnage de « Madame la présidente ». 
Le Premier ministre du pays est une femme et la meilleure amie de Fitina. Elle est aussi le Ministre du Pétrole et des Hydrocarbures, des Infrastructures et de l'Economie et des Finances. Elle est surnommée « le ministre des postes juteux » (p. 67). Pour mériter ce poste, elle doit être le bras-droit de Fitina. Mais, nous reconnaissons qu'il existe un grand décalage ici, au niveau de la caractérisation. Qui est le Premier ministre ? Comment s'appelle-t-elle ? Quel est le nom de sa famille ? La romancière est silencieuse sur ce personnage car il y a trop d'attention mise sur le personnage principal de Fitina. Cette dame n'a pas de nom propre malgré le fait qu'elle a une famille qui vint rechercher son corps à sa mort. C'est une omission grave car les autres personnages féminins ont des noms propres.

Tassouma est la cousine éloignée de Fitina. C'est elle qui remplace le Colonel Kèléfa en qualité de cheffe de la garde présidentielle (p. 201). Sa durée d'apparition est courte suite la disparition de Kèléfa le frère de Fitina. Elle est fidèle à Fitina jusqu'à la mort car elle participe à la guerre contre les fantômes en défendant la présidente. Pour pouvoir participer à cette bataille, elle devrait être une femme forte et fortifiée de la magie africaine.

Kandji est une étudiante qui crée et dirige le Syndicat des Etudiants Révoltés (SER) mais qui finit par céder au pouvoir de Fitina à cause de l'argent. Elle devient le bras séculier de Fitina (p. 129). Elle est lesbienne et dirige le groupe opposé après avoir déclaré son soutien à Fitina. Elle a contribué à la dictature de Fitina et aux manifestations estudiantines sur le campus. Elle a des parents qui assuraient ses frais de scolarité mais elle vit sans en avoir besoin car le gouvernement de Fitina assurait son train de vie hebdomadaire.

Kriza est la jeune dame jolie et attirante envoyée spécialement pour séduire le juge qui refuse d'être corrompu par le gouvernement de Fitina, la présidente.

Mme N'ko est l'épouse du juge que Kriza a essayé de séduire. Elle organise clandestinement sur les réseaux sociaux une dénonciation de l'affaire de Kriza et la déformation du personnage de son mari.

Les autres personnages féminins sont Sophie, les vieilles dames qui ont participé à la marche de la manifestation, les jeunes étudiantes violées et la dame enceinte sacrifiée qui donne naissance à l'enfant retrouvé par le féticheur, Djomori. La romancière n'a pas consacré assez de temps pour développer ces personnages féminins.

\section{Les personnages masculins}

Parmi les personnages masculins importants nous trouvons les deux frères de Fitina, Kèléfa et Kotigui. Kèléfa est militaire (p. 24). Il fut nommé au grade de colonel et sera affecté à la tête de la garde présidentielle (p. 70). Il s'exile lorsqu'il prend connaissance que leur sœur bien aimée voulait sacrifier l'un d'eux.

Kotigui est le plus aimé des frères car c'est lui qui est chargé de consulter des sorciers pour protéger sa sœur. Il croit fortement à la sorcellerie, au fétichisme et à la magie noire. C'est grâce à lui que sa sœur devient une personnalité forte en mysticisme. Il remplace Madame le Premier ministre. C'est lui qui aurait dû délivrer sa sœur par le sacrifice de son sang, mais lui et son frère militaire s'exilent en quittant le pays. 
Djomori est le grand féticheur de Madame la présidente. C'est « un très beau jeune homme au teint clair, vêtu à la dernière mode d'une chemise et d'un pantalon noir avec des cheveux lisses et longs, retenus par un petit élastique noir » (p. 50). Appelée 'le puissant' et 'l'incommensurable' (p. 51), il se transforme lorsqu' il commence les consultations mystiques (p. 52). Ce féticheur est un homme mystérieux dont la romancière a consacré assez de temps et de pages à décrire et à narrer. Il ne meurt pas à la fin mais change d'environnement en quittant son endroit mystérieux pour aller revivre quelque part. Il ne meurt pas mais son client principal meurt. Ses mains sont bien salies du sang des innocents utilisés pour des sacrifices.

M. Banoussa, le Directeur de l'Agence des Investissements économiques est comptable et le neveu de Madame le Premier ministre (p. 77). Un homme corrompu, qui détourne l'argent du pays et prend frauduleusement l'argent mis à la disposition du pays après avoir causé l'incendie du marché central. Il fut arrêté, jugé et condamné à la prison puis relâché plus tard à cause de sa relation avec madame le Premier ministre.

M. N'ko est un juge et un homme droit qu'il est difficile de corrompre. On voit le mélange de la justice et de la politique. Il est séduit par Kriza, une jeune dame jolie et attirante envoyée spécialement pour le séduire. Grâce à sa résistance, l'affaire déclenche une réaction sur les réseaux sociaux organisée clandestinement par sa femme, Mme N'ko.

Nataba, l'ancien leader de l'opposition politique devenu vice-président de Fitina, a vendu sa conscience. Il remplace aussi Kotigui au poste qu'occupait le frère de Fitina, lorsque celui-ci quitte le pays. Il devient le troisième premier ministre. Nataba est comme les politiciens contemporains africains qui se prostituent d'une partie politique à un autre en fonction du parti au pouvoir.

Djalo remplace le leader Nataba qui trahit le parti en déclarant son soutien et en acceptant la position de vice-président de pays. Le nouveau leader fut Djalo. Il est tué pour son opposition et fut mangé par les invités de la présidente Fitina à leur insu. Le cannibalisme est ardent ici. C'est lui qui dirige les morts ou les fantômes pour combattre Fitina afin de sauver le peuple du Louma.

Simakan est le président sortant du pays. On ne connait rien de lui. Il cède la place pour l'introduction des huitièmes élections présidentielles ayant accompli son mandat dont la romancière ne dit rien.

Les autres personnages sont Roger, Kafana, Alex, Sylvestre, Fidèle, Anoblé, Xavier et la population estudiantine, les vieux et les vieilles et les enfants. On ne peut pas oublier les journalistes, les militaires, etc.

\section{Les personnages mystérieux/fantômiques}

Les personnages fantômiques sont de genre mixte. Tous les personnages mystérieux qui sont aussi des fantômes étaient des personnages masculins ou féminins vivants déjà décrits. Ils deviennent fantômes et forment le Syndicat des Fantômes et ce sont eux qui ont tous participé à la grande bataille contre Fitina et ses gardes pour délivrer le pays Louma de la dictature fitinaise. Le genre romanesque du fantastique ressemble aux romans de Sony Labou Tansi (1979, 1981). La combination de tous ces personnages donne un style unique au roman de Fanny-Cissé. 


\section{Le style}

Le style de l'auteur est simple à part les africanismes inexpliqués. Le langage est un langage simple et direct mais remplie d'africanismes pour lesquels parfois le lecteur sera obligé de chercher leur signification. Mais, le plus souvent, le lecteur poursuivra sans s'engager dans des contextes explicatifs et des contextes définitoires quasi inexistants qui auraient nui à la lecture et grâce aux inférences contextuelles, on pourra tirer des sens de certains africanismes ainsi que des néologismes. Pour mieux faciliter la compréhension de cette étude critique, nous mettons ci-dessous un découpage des chapitres dans le roman.

Le découpage des pages selon les chapitres nous donne, à partir de la représentation graphique, le début romanesque avec le commencement du pouvoir de Fitina et son ascension à l'apogée de son pouvoir et sa dictature avant la descente et la chute au chapitre 6 . A ce chapitre se trouve le mélange de la justice et de la politique avec l'affaire de la séduction du juge, N'ko. Six chapitres après ceci, c'est-à-dire au douzième chapitre, on voit une mauvaise descente et une chute grave avec le lancement des roquettes qui auraient dû assassiner la présidente, un coup d'état échoué. Le peuple est mécontent avec l'échec de l'attentat et ce qui suit c'est l'émeute incessante du peuple.

Grâce à l'intervention surnaturelle, le pays témoigne de la destruction de la femme dictateur et le pays reprend haleine. Il sera nécessaire de donner des résumés chapitre par chapitre afin de mieux comprendre l'analyse du roman (figure 1 et 2).

\section{Analyse des chapitres}

Chapitre 1 contient 14 pages et énonce une nouvelle génération de mendiants. Les pays voisins de Louma ainsi que ce pays ne sont pas prêts ou mûrs pour la démocratie (p. 10). En parlant de Louma, la narratrice fait une généralisation du continent auquel appartient ce pays. Disons que la romancière ressemble à Ahmadou Kourouma (1970) par son appellation de «bâtard » pour désigner le pays : « La République de Louma était donc à un stade intermédiaire que certains n'auraient pas hésité à qualifier de «bâtard » ou d'hybride parce qu'elle n'était plus au stade du parti unique, mais qu'elle n'était pas encore réellement démocratique » (pp. 10-11). Le président sortant s'appelle Simakan (p. 14); celui-ci cède la place pour l'introduction des huitièmes élections présidentielles ayant une quarantaine de candidatures parmi lesquelles figure une candidature féminine donnant un courage exceptionnel dans l'arène politique. Elle s'appelle Fitina. Elle est femme célibataire et sans enfant.

Dans le chapitre 2, la narration et la description effectuées sont si vivantes que c'est l'un des deux chapitres le plus long parmi les 15 chapitres du roman. Il contient 20 pages. Ce chapitre présente une narration et une description minutieuses de la sorcellerie et du fétichisme. Toute la sorcellerie et le fétichisme sont basés sur les élections présidentielles du pays Louma. Ce chapitre prépare les lecteurs sur les disparitions des gens (des enfants uniques, des albinos, des gens célèbres, des femmes enceintes) pour des sacrifices rituels, surtout des sacrifices humains 


\begin{tabular}{|c|c|c|}
\hline Chapitres & Pages & Nombre total de pages \\
\hline 1 & $7-20$ & 14 \\
\hline 2 & $21-40$ & 20 \\
\hline 3 & $41-56$ & 16 \\
\hline 4 & $57-72$ & 16 \\
\hline 5 & $73-92$ & 20 \\
\hline 6 & $93-104$ & 12 \\
\hline 7 & $105-121$ & 17 \\
\hline 8 & $122-138$ & 17 \\
\hline 9 & $139-153$ & 15 \\
\hline 10 & $154-169$ & 16 \\
\hline 11 & $170-180$ & 11 \\
\hline 12 & $181-190$ & 10 \\
\hline 13 & $191-204$ & 14 \\
\hline 14 & $205-216$ & 12 \\
\hline 15 & $217-227$ & 11 \\
\hline
\end{tabular}

Fig. 1 Découpage des chapitres et nombre total des pages

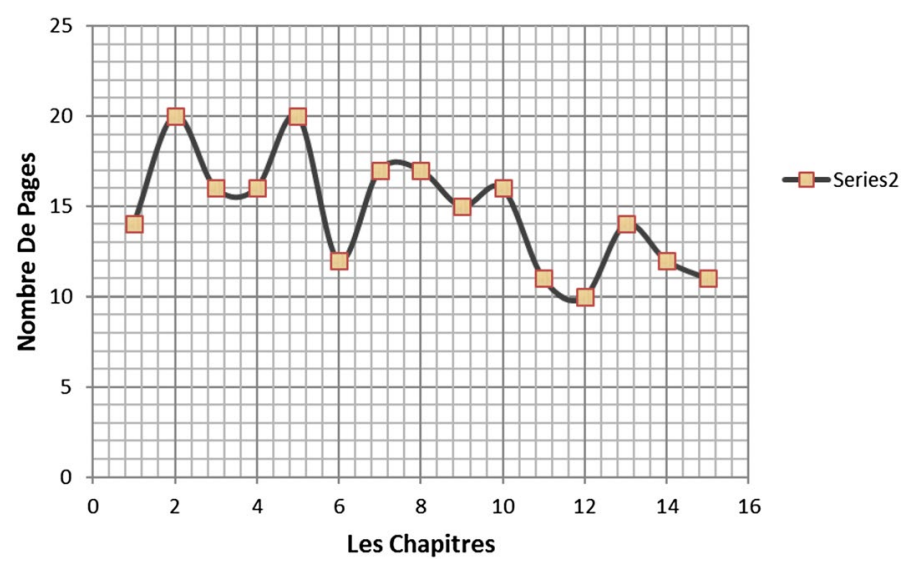

Fig. 2 Représentation graphique des chapitres et des pages démontrant la croissance ou la décroissance du nombre total des pages

pour garantir la victoire des candidats. On nous présente l'entrée de Djomori, le puissant et l'incommensurable, le soutient de Fitina. Ce chapitre est une sorte de prolepse aux évènements que nous verrons plus tard dans les chapitres qui suivent. Il énonce et explique les phénomènes sur des organes prélevés, des 
talismans, des personnes enlevées, des parties intimes du corps humain à travers l'intervention du frère de Fitina, le protagoniste. L'explication de comment déféquer les sorts, déjouer le sort lancé par les excréments, urine, etc., (p. 35). A travers ce chapitre, on peut comprendre l'utilisation des carrefours, des canaris, des «colas expiatoires du mal » (p. 37) ainsi que l'importance et le rôle des mendiants. D'ici, on peut faire une comparaison avec les mendiants dans La grève des battù (1979) d'Aminata Sow Fall, et la critique d'Emokpae-Ogbebor sur la mendicité du même roman. Fanny-Cissé comme Aminata Sow Fall réussit à montrer non seulement le rôle indispensable des mendiants mais la typologie des mendiants. La romancière peut donc se comparer à Fall où l'on remarque des mendiants à Abidjan comme à Dakar.

Le chapitre 3 qui a pris 16 pages parle de la campagne électorale avec ses ambiances festives et les promesses électorales des candidats. Les promesses incluent la lutte contre la corruption, le harcèlement sexuel dans les écoles, collèges et lycées (p. 44) et contre les chantages sur les professeurs. C'est à l'apogée des campagnes électorales que nous sommes introduits dans la destination mystérieuse du grand féticheur, la forêt sacrée de Djomori. La description écologique de cette ambiance donne un certain symbolisme mystérieux et mystique avec les arbres immenses comme des baobabs, l'oiseau mystérieux comme le hibou, les animaux aussi mystiques en Afrique comme les chats et le féticheur même (pp. 49-52). Nous voyons la description du féticheur, Djomori et son milieu tel que sa maison (p. 49). Le phénomène de maraboutage dans ce chapitre nous rappelle d'El Hadji Kader Bèye dans Xâla de Sembène, de Les soleils des indépendances et Les Votes des bêtes sauvages de Kourouma et le féticheur dans ses œuvres, d'Aminata Sow Fall dans La Grève des battù (1979) et Le Revenant (1976). Pour pouvoir devenir la présidente de son pays, Fitina, la seule candidate féminine doit remplir trois exigences du féticheur puissant à savoir:

1. Don de sa fertilité (la fertilité est un élément important pour l'homme et surtout une femme africaine)

2. Vœu de célibat (refuser de se marier pour une femme, dans la société africaine est un grand sacrifice)

3. Don de la vie d'un albinos (un sacrifice humain qui sera périodiquement renouvelé pour maintenir l'efficacité des protections faites (p. 54)).

Le chapitre 4 contient 16 pages de narration comme le chapitre précédent. Il s'agit de la description minutieuse des résultats de la consultation avec Djomori, le puissant féticheur de Fitina. On y voit aussi la récompense de Djomori qui se trouve dans les conditions qu'il a imposées à Fitina. Elle reçoit une valisette contenant la tête d'un célèbre chanteur albinos (p. 60), une grosse bague, une grosse amulette cousue, une autre amulette en forme de ceinture sur laquelle pendait une centaine de minuscules cauris, un gri-gri noir, et des incantations à prononcer ainsi que son totem. Fitina remporta la victoire et son investiture eut lieu. Elle commence avec le népotisme. "Les trois quarts du gouvernement étaient constitués par des membres de la famille élargie ou par des connaissances proches de 
la présidente Fitina » (p. 67). Il y a un « nombre incalculable de conseillers payés $\gg($ p. 71).

Le chapitre 5 contient 20 pages comme le chapitre 2. C'est l'un des deux chapitres qui sont les plus longs chapitres du roman. Ce chapitre présente le gouvernement de Madame la Présidente et l'incendie du marché central. C'est une révélation de la corruption du gouvernement car l'incendie devient « une manière de gagner frauduleusement de l'argent » (p. 75). Le directeur de l'Agence des investissements économiques, M. Banoussa, le neveu de Madame le Premier ministre est corrompu et exploite les masses populaires. C'est une exploitation des commerçantes et de la masse populaire et la souffrance du peuple.

Le chapitre 6 est composé de 12 pages après les 20 pages du chapitre précédent. C'est une diminution forte des évènements romanesques. On note les intrigues de Madame la Présidente et ses jeux politiques. Dans ce chapitre, on voit comment Fitina a réussi à déjouer Nataba, le leadeur de l'opposition et le nomme son viceprésident (p. 94). Ensuite, chaque personne qui ose s'opposer à elle est, soit éliminée complètement, soit détournée ou déjouée. M. N'Ko, le juge droit a été séduit par Kriza, une jeune dame séduisante car il défit la présidente en envoyant M. Banoussa en prison puisque celui-ci est coupable. Il s'agit ici de la justice et de la politique. Fitina s'égare et 50 hommes de Dieu essaie de lui en parler mais elle refuse d'accepter leur conseil. (p. 103).

Le chapitre 7 contient 17 pages et est basé sur la formation du Syndicat des Etudiants Révoltés (SER) et leurs activités contre le gouvernement. La meneuse des troupes estudiantines s'appelle Kandji, elle dirige les grèves estudiantines et finit par travailler pour le gouvernement à cause de l'argent. C'est l'affaire du « marché de Banoussa » et « le scandale du Tribunal » qui mènent tous les deux à l'établissement des réseaux sociaux et la restriction de l'accès aux réseaux sociaux par le gouvernement de Madame la Présidente, Fitina. Mais, le Pouvoir conclut que les étudiants étaient manipulés et interdit les réseaux sociaux ainsi que des grèves sur le campus (p. 111).

Le chapitre 8 contient 17 pages au total et est basé sur les manifestations estudiantines. Kandji démissionne du Syndicat des Etudiants Révoltés (SER) et devient le bras séculier de Fitina car l'argent avait grisé ses mains (p. 129). C'est l'établissement des guérillas universitaires entre le SER et le camp de Kandji qui travaille maintenant pour le gouvernement. Le Pouvoir fait venir les militaires sur le campus et ils violent les étudiantes. C'est la descente militaire (p. 134). La dictature montante et rampante (pp. 135, 136) respectivement se voit.

Le chapitre 9 contient 15 pages. Fitina est obligée de rendre visite à son féticheur, Djomori pour résoudre les problèmes estudiantins. Elle sera obligée d'enterrer des fétiches sur le campus pour rétablir la paix. Le leadeur du parti opposé est remplacé par Djalo qui disparait un jour car la présidente a fait venir deux lions au sous-sol du palais présidentiel pour dévorer ses ennemis. Mais avant le commencement de cette « dictature sans nom », elle commence à pratiquer le cannibalisme puisque l'opposant, Djalo fut consommé par tous ses invités à un dîner présidentiel (p. 150).

Le chapitre 10 est composé de 16 pages. Le comportement de la présidente devient sauvage et antidémocratique (p. 156) car tous ceux qui osent l'affronter d'une manière ou d'une autre finissent par être tué tel son psychiatre venu d'Europe 
(p. 152). La tentative de son premier ministre de l'empoisonner n'a pas marché (pp. 157-158). Ensuite, l'effort de son médecin personnel est voué à l'échec (pp. 161-162). Ces deux coupables ont été oferts à manger aux lions féroces, ainsi que son garde du corps qui a tiré à plusieurs reprises sur la présidente (p. 167). Avec tous ces évènements en peu de temps, Fitina sera obligée de consulter Djomori le puissant féticheur pour rétablir le calme dans le pays et détourner toute la haine contre sa personnalité. Voilà que « la dernière année du mandat de la présidente Fitina s'écroule rapidement afin que le pays change enfin main » mais Fitina n'est pas prête à quitter et à céder le pouvoir.

Le chapitre 11 est composé de 11 pages. Il est marqué par une autre descente. Fitina ni ne veut pas changer ni ne veut quitter le pouvoir. Le peuple s'inquiète. Malheureusement, Fitina veut modifier la constitution du pays à sa guise afin de devenir «Présidente à vie » (p. 173). La population est provoquée hors de sa capacité et il en résulte des manifestations des vieux et des jeunes enfants, qui, malheureusement furent battus, produisant des blessées et des morts. C'est le plus provoquant des affronts du pouvoir de Fitina, Madame la présidente.

Le chapitre 12 est le plus court des chapitres avec 10 pages. C'est encore une descente en enfer. C'est un coup d'état qui n'a pas réussi. Cet attentat échoué est un message clair et direct à la présidente qu'elle doit quitter le gouvernement car le peuple est mécontent envers elle et son gouvernement. Deux roquettes atterrissent l'une après l'autre dans la chambre de Fitina (p. 182). La Radiodiffusion loumaise a été aussi attaquée mais sans succès. L'armée loumaise fait partie de ce coup d'Etat échoué. On croyait que ce serait la fin de Fitina mais grâce à ses forces mystérieuses et mystiques, elle a survécu à tous ces attentats. Mais, pour les lecteurs, c'est déjà la fin de cette présidente car la population n'est pas du tout contente d'elle.

Les évènements du chapitre 13 s'étendent sur 14 pages. Le peuple essaie de reprendre vie. C'est un chapitre caractérisé par des émeutes du peuple partout dans le pays. C'est la prise de conscience du peuple car c'est un grand défi pour le peuple que Fitina ait eu besoin de sacrifier son frère préféré, Kotigui « pour conserver son pouvoir » (p. 196). Est-ce que cela va marcher ? Ses deux frères fuirent le pays en l'abandonnant à son sort. Elle sera donc obligée de faire un sacrifice rituel d'une femme enceinte vivante. Le renouvèlement des pouvoirs mystiques fait prolonger ce chapitre car les forces surnaturelles interviennent et viennent à l'aide des vivants impuissants. En Afrique, les morts ne sont pas morts. Il y eut une conférence nationale tenue par les morts devenus des fantômes (p. 202) et Fitina eut une séance fantômique dans son rêve (p. 203). Ces fantômes forment le «Syndicat des Fantômes » (p. 204) ayant la mission de «mettre fin à la dictature de la présidente Fitina » (p. 203) afin de délivrer le pays loumaise.

Dans le chapitre 14 qui compte 12 pages, se déroule le combat entre Fitina et les fantômes au sous-sol du Palais présidentiel (p. 207). Le grand féticheur, Djomori ne comprenait plus ce qui se passait car tout le dépassait. Les interventions surnaturelles dépassent même le grand féticheur. Donc, Fitina devait prendre elle-même son sort en main et devait faire face aux fantômes. Les fantômes rendirent d'abord visite à Djomori et causèrent des dégâts à l'aide du tonnerre qui délogea tous les animaux, les oiseaux et les arbres au domicile du grand féticheur. Les fantômes vainquirent la garde mystique présidentielle de Tassouma et ses membres (pp. 212-213). 
Voyant ses gardes exterminées, Fitina chargea contre les fantômes dirigés par le feu Djalo. Elle fut abattue et fut dévorée par ses lions. «Ainsi, Fitina, qui avait fait de ses lions une arme de dissuasion, avait-elle fini dévorée par eux » (p. 216). C'est la fin de Fitina et la fin de la dictature.

Le dernier chapitre contient 11 pages de clôture. La descente, cette fois, dans la vallée, plonge le pays dans une incertitude malgré le fait que l'on s'est déjà débarrassé de la 'dictateuse'. La population est pleine de joie et il y eut une manifestation joyeuse partout dans le pays de Louma. Le désordre qui caractérise cette descente est rétablie par un religieux charismatique qui appela le président de la Cour suprême à assurer l'intérim pendant trois mois avant d'organiser des élections transparentes.

C'est étonnant de voir la survivre le grand féticheur qui s'est repenti par la naissance mystérieuse du bébé de la femme enceinte sacrifiée et enterrée. Le bébé présumé mort était né. La naissance et la survivance mystérieuse du bébé incarne une nouvelle vie pour la population et le pays. Kèléfa et Kotigui, les frères de Fitina ainsi que d'autres exilés du pays retournent au pays. C'est le chapitre de réparation et de restauration. Alors, il y a de l'avenir aux évènements politiques néfastes malgré les dirigeants et les gouvernements en question.

\section{Les esthétiques et les critiques du roman}

Pour le moment, il n'existe aucune critique sur le roman. La critique de la politique partout dans le monde n'est pas facile. C'est une chose qui a besoin du courage de la part de l'écrivain. La plupart des écrivains ont trouvé la mort à cause de leur engagement politique. C'est la raison pour laquelle certains écrivains se servent de pseudonymes.

\section{Conclusion}

Ce roman contemporain est rempli de mysticisme et de dictature inconnue au pays imaginaire. Le féminisme projeté avec une protagoniste féminine et d'autres personnages féminins constitués en des rôles fortiiés échouent tous dans leurs rôles. La présidente, le premier ministre féminin, la présidente du syndicat estudiantin et la garde personnelle de la présidente rencontrent toutes un grand échec. Les féministes mondiaux sont déçues de voir que le roman autrement constitué de force féministe sont toutes garnies d'une forte négativité. La politique féminisée ne s'achèvet-elle pas de phénomènes positifs au moins pour renforcer la présence de la femme mondialement?

Les personnages romanesques ont un seul nom et la plupart d'entre eux n'ont pas de nom de famille et certains n'ont pas de nom tel Madame le Premier ministre, les journalistes, d'autres étudiants. Néanmoins, c'est un roman à couper le souffle à cause de la description mystique et mystérieuse des personnages et des évènements. Le roman se ferme sur une note positive, un avenir positif où il $\mathrm{y}$ a une restauration et un meilleure future. 
Open Access This article is licensed under a Creative Commons Attribution 4.0 International License, which permits use, sharing, adaptation, distribution and reproduction in any medium or format, as long as you give appropriate credit to the original author(s) and the source, provide a link to the Creative Commons licence, and indicate if changes were made. The images or other third party material in this article are included in the article's Creative Commons licence, unless indicated otherwise in a credit line to the material. If material is not included in the article's Creative Commons licence and your intended use is not permitted by statutory regulation or exceeds the permitted use, you will need to obtain permission directly from the copyright holder. To view a copy of this licence, visit http://creativecommons.org/licen ses/by/4.0/.

\section{Références}

Adejir, T. (2000). Political disillusionment and aesthetic evolution in the novels of Alioum Fantouré. In S. A. Ojo \& O. Oke (Eds.), Themes in African literature in French: A collection of essays (pp. 117-139). Ibadan: Spectrum Books Limited.

Dogbé, Y.-E. (1979). La Victime. Le Mé-Sur-Seine: Editions Akpagnon.

Elaho, R. O. (2000). Socio-political consciousness in the novels of Yves-Emmanuel Dogbé. In S. A. Ojo \& O. Oke (Eds.), Themes in African literature in French: A collection of essays (pp. 141-152). Ibadan: Spectrum Books Limited.

Emokpae-Ogbebor, O. (2017). La Dynamique des genres dans La Vie et demie et L'Etat Honteux de Sony Labou Tansi. RANEUF, 15, 19-38.

Fanny-Cissé, F. (2015). Madame la présidente. Abidjan: NEI-CEDA.

Fantouré, A. (1972). Le Cercle des tropiques. Paris: Présence Africaine.

Fatunde, T. (2002). La Calebasse cassée. Ibadan: Bookcraft Ltd.

Kourouma, A. (1970). Les Soleils des indépendances. Paris: Editions du Seuils.

Sow-Fall, A. (1976). Le Revenant. Dakar: N.E.A.

Sow-Fall, A. (1979). La Grève des battù. Dakar-Abidjan-Lomé: N. E. A.

Tansi, S. L. (1979). La Vie et demie. Paris: Seuil.

Tansi, S. L. (1981). L'Etat honteux. Paris: Seuil.

Publisher's Note Springer Nature remains neutral with regard to jurisdictional claims in published maps and institutional affiliations. 